PROCEEDINGS OF THE

AMERICAN MATHEMATICAL SOCIETY

Volume 132, Number 6, Pages 1713-1720

S 0002-9939(04)07224-7

Article electronically published on January 7, 2004

\title{
A NOTE ON COMMUTATIVITY UP TO A FACTOR OF BOUNDED OPERATORS
}

\author{
JIAN YANG AND HONG-KE DU
}

(Communicated by Joseph A. Ball)

\begin{abstract}
In this note, we explore commutativity up to a factor $A B=\lambda B A$ for bounded operators $A$ and $B$ in a complex Hilbert space. Conditions on possible values of the factor $\lambda$ are formulated and shown to depend on spectral properties of the operators. Commutativity up to a unitary factor is considered. In some cases, we obtain some properties of the solution space of the operator equation $A X=\lambda X A$ and explore the structures of $A$ and $B$ that satisfy $A B=\lambda B A$ for some $\lambda \in \mathbb{C} \backslash\{0\}$. A quantum effect is an operator $A$ on a complex Hilbert space that satisfies $0 \leq A \leq I$. The sequential product of quantum effects $A$ and $B$ is defined by $A \circ B=A^{\frac{1}{2}} B A^{\frac{1}{2}}$. We also obtain properties of the sequential product.
\end{abstract}

\section{INTRODUCTION}

Commutation relations between selfadjoint operators in a complex Hilbert space play an important role in the interpretation of quantum mechanical observables and analysis of their spectra. For related works refer to [2], 3], 4], [6], [8], [9] and [12. Accordingly, such relations have been extensively studied in the mathematical literature (see, for example, the classic study of C. R. Putnam in [11). An interesting, related aspect concerns the commutativity up to a factor for a pair of operators. Certain forms of non-commutativity can be conveniently phrased in this way. In [1, J. A. Brooke, P. Busch and D. B. Pearson gave some examples to illustrate this. A quantum effect is a yes-no measurement. An effect is represented by an operator $A$ on a Hilbert space that satisfies $0 \leq A \leq I$. A sharp effect is represented by a selfadjoint projection operator on a Hilbert space. The sequential product of quantum effects $A$ and $B$ is defined by $A \circ B=A^{\frac{1}{2}} B A^{\frac{1}{2}}$. Sequential measurements are very important in quantum mechanics. For detailed works refer to 8], 9] and [12. Let $H$ be a complex Hilbert space, $B(H)$ be the Banach algebra of bounded linear operators on $H, \varepsilon(H)$ be the set of quantum effects on $H, P(H)$ be the set of sharp effects on $H, I$ be the identity operator on some Hilbert space and $M_{n \times m}$ be the set of $n \times m$ matrices. For an operator $A \in B(H)$, denote by $N(A), R(A), \sigma(A), r(A)$ the null space, the range, the spectra and the spectral radius of $A$, respectively; $\operatorname{dim} N(A)$ denotes the dimension of $N(A)$. Recall that, for

Received by the editors October 25, 2002 and, in revised form, January 9, 2003.

2000 Mathematics Subject Classification. Primary 47A10.

Key words and phrases. Hilbert space, commutator, selfadjointness, normal operator, quantum effect.

This work was partially supported by the National Natural Science Foundation of China. 
$A, B \in B(H), A$ and $B$ commute up to a factor means that $A B=\lambda B A$, for some $\lambda \in \mathbb{C} \backslash\{0\}$, and $A$ and $B$ commute up to a unitary factor means that $A B=U B A$, where $U$ is a unitary operator in $B(H)$. For each $0 \leq A \in B(H), B \in B(H)$, we also define $A \circ B=A^{\frac{1}{2}} B A^{\frac{1}{2}}$. The main results shown by J. A. Brooke, P. Busch and D. B. Pearson in [1] are the following two theorems.

Theorem 1.1 ([1]). Let $A, B \in B(H)$ such that $A B=\lambda B A \neq 0, \lambda \in \mathbb{C}$. Then

(i) if $A$ or $B$ is selfadjoint, then $\lambda \in \mathbb{R}$;

(ii) if both $A$ and $B$ are selfadjoint, then $\lambda \in\{-1,1\}$; and

(iii) if $A$ and $B$ are selfadjoint and one of them is positive, then $\lambda=1$.

Theorem 1.2 ([1]). Let $A, B \in B(H)$ be selfadjoint operators. The following statements are equivalent.

(i) $A B=U B A$ for some unitary operator $U$.

(ii) $A B^{2}=B^{2} A$ and $B A^{2}=A^{2} B$.

In 9] S. Gudder and G. Nagy gave the following result on sequential measurement.

Theorem $1.3([9])$. For $A, B \in \varepsilon(H)$, if $A \circ B \in P(H)$, then $A B=B A$.

They put forward an open problem: if $A, B \in \varepsilon(H)$ with $\operatorname{dim} H=\infty$ and $A \circ B \geq B$, does $A B=B A=B$ hold?

In this paper, firstly we give simple proofs of the theorems above and generalizations of them. Secondly, we show a further relation between the spectra of $A B$ and the factor $\lambda$. In the case that $H$ is finite dimensional, we obtain a property of the solution space of the operator equation $A X=\lambda X A$. Also, if $A$ has finite rank and is normal, we explore the structure of $A$ and $B$ which commute up to a factor. Thirdly, we give a generalization of Theorem 1.3 in [9] with proof different from [9] and answer the open question raised by S. Gudder and G. Nagy. This question was also independently answered by A. Gheondea and S. Gudder in [7].

We will use repeatedly the Fuglede-Putnam Theorem.

Theorem 1.4 (Fuglede-Putnam Theorem ([11)). If $N$ and $M$ are normal operators on $H$ and $K$, and $B: K \rightarrow H$ is an operator such that $N B=B M$; then $N^{*} B=B M^{*}$.

\section{Pairs COMmUting up tO A FACTOR}

Theorem 2.1. Let $A, B \in B(H)$ such that $A B=\lambda B A \neq 0, \lambda \in \mathbb{C}$. Then

(i) if $A$ or $B$ is selfadjoint, then $\lambda \in \mathbb{R}$;

(ii) if either $A$ or $B$ is selfadjoint and the other is normal, then $\lambda \in\{-1,1\}$; and

(iii) if both $A$ and $B$ are normal, then $|\lambda|=1$.

Proof. (i) Suppose that $A$ is selfadjoint; then $\lambda A$ is normal. By the Fuglede-Putnam Theorem, we have $A B=\bar{\lambda} B A$. Hence $(\lambda-\bar{\lambda})=0$. This implies $\lambda \in \mathbb{R}$.

(ii) Suppose that $A$ is normal and $B$ is selfadjoint. By the Fuglede-Putnam Theorem, we have $A^{*} B=\bar{\lambda} B A^{*}$. Then $A A^{*} B=\bar{\lambda} A B A^{*}=|\lambda|^{2} B A A^{*}$. From (i) and (iii) of Theorem 1.1, we have $\lambda \in \mathbb{R}$ and $|\lambda|^{2}=1$. Hence $\lambda \in\{-1,1\}$.

(iii) From $A B=\lambda B A$, we have $B^{*} A^{*}=\bar{\lambda} A^{*} B^{*}$. By the Fuglede-Putnam Theorem, we get $A^{*} B=\bar{\lambda} B A^{*}$ and $B A^{*}=\lambda A^{*} B$. Hence $A^{*} B=|\lambda|^{2} A^{*} B$. Since $A B \neq 0$ and $A$ is normal, we can get $A^{*} B \neq 0$. Therefore $|\lambda|=1$. 
In fact, with similar deduction, we can generalize (i) of Theorem 2.1 in the following way:

Corollary 2.2. Suppose $A, B, C \in B(H)$ and $A, C$ are selfadjoint operators with $A B=\lambda B C \neq 0, \lambda \in \mathbb{C}$; then $\lambda \in \mathbb{R}$.

The following result is a generalization of Theorem 1.2 in [1].

Theorem 2.3. Let $A, B \in B(H)$. Then the following statements are equivalent.

(i) $A B B^{*}=B^{*} B A$ and $B A A^{*}=A^{*} A B$.

(ii) Both $A B$ and $B A$ are normal.

(iii) There exist unitary operators $U$ and $V$ in $B(H)$ such that $A B=U B^{*} A^{*}$ and $B A=V A^{*} B^{*}$.

Proof. The equivalence of (i) and (ii) was proved by S. Gudder and G. Nagy in [8].

(ii) $\Rightarrow$ (iii) By the fact that an operator $N \in B(H)$ is normal if and only if there exists a unitary operator $U$ such that $N=U N^{*}$, the proof is trivial.

(iii) $\Rightarrow$ (ii) Observe that $B^{*} A^{*}=A B U^{*}$, and so $A B=U A B U^{*}$. Hence $A B$ commutes with $U$ and $U^{*}$, and similarly for $B^{*} A^{*}$. Thus we get $A B B^{*} A^{*}=$ $U B^{*} A^{*} U^{*} A B=B^{*} A^{*} U U^{*} A B=B^{*} A^{*} A B$. Hence $A B$ is normal. Similarly, from $B A=V A^{*} B^{*}$, we can get $B A$ is normal.

If both $A$ and $B$ are selfadjoint, then Theorem 2.3 becomes Proposition 4.1 and Proposition 4.2 in 1 .

For given $0 \neq A \in B(H)$, let

$$
S_{A}^{n}=\{B \in B(H): \sigma(A B) \text { has exactly } n \text { distinct nonzero values }\} .
$$

Then we have

Theorem 2.4. For each $0<n<\infty$ and $B \in S_{A}^{n}$, if $A B=\lambda B A$ for some $\lambda \in \mathbb{C}$, then $\lambda^{n}=1$.

Proof. Suppose $B \in S_{A}^{n}$ with $A B=\lambda B A$. Let $\sigma(A B) \backslash\{0\}=\left\{\alpha_{1}, \cdots, \alpha_{n}\right\}$. Then $\left\{\alpha_{1}, \cdots, \alpha_{n}\right\}=\left\{\lambda \alpha_{1}, \cdots, \lambda \alpha_{n}\right\}$, since $\sigma(A B) \backslash\{0\}=\sigma(B A) \backslash\{0\}$. Clearly, for each $\alpha_{i_{1}} \in \sigma(A B) \backslash\{0\}$, there exist $1 \leq p \leq n$ and $\alpha_{i_{j}} \in \sigma(A B) \backslash\{0\}, 1<j \leq p$, with $\alpha_{i_{k}} \neq \alpha_{i_{l}}, 1<k \neq l \leq p$, such that $\alpha_{i_{1}}=\lambda \alpha_{i_{2}}=\cdots=\lambda^{p-1} \alpha_{i_{p}}=\lambda^{p} \alpha_{i_{1}}$. If $\alpha_{i_{1}}=\lambda \alpha_{i_{2}}=\cdots=\lambda^{p-1} \alpha_{i_{p}}=\lambda^{p} \alpha_{i_{1}}$ and $\alpha_{j_{1}}=\lambda \alpha_{j_{2}}=\cdots=\lambda^{q-1} \alpha_{j_{q}}=\lambda^{q} \alpha_{j_{1}}$, for distinct $i_{1}$ and $j_{1}$, then $p=q$ and $\alpha_{i_{l}} \neq \alpha_{i_{k}}, 1<l, k \leq p$. In fact, we assume that $p<q$. Then $\lambda^{p}=\lambda^{q}=1$ and $\lambda^{q-p}=1$; hence $\alpha_{j_{1}}=\alpha_{j_{q-p+1}}$. It is a contradiction. Then we have $\frac{n}{p} \in \mathbb{N}$. Hence $\lambda^{n}=1$.

The next result gives some restrictions that must be satisfied by a pair of operators commuting up to a scalar factor.

Theorem 2.5. Let $A, B \in B(H)$ such that $A B=\lambda B A \neq 0$, for some $\lambda \in \mathbb{C}$. Then

(i) $A B$ is bounded below if and only if both $A$ and $B$ are bounded below;

(ii) if $A$ is normal and $R(B)$ is dense, then $A B$ is not nilpotent.

Proof. (i) If $A$ and $B$ are bounded below, clearly, we have that $A B$ is bounded below. Conversely, if $A B$ is bounded below, then $B$ is bounded below. We also have that $A$ is bounded below, since $A B=\lambda B A$.

(ii) If $A B=\lambda B A$ and $A$ is normal, then $N(A)$ and $\overline{R(A)}$ are reducing subspaces of $A$ and $B$. Thus $A$ and $B$ have forms $A=\operatorname{diag}\left(A_{1}, 0\right)$ and $B=\operatorname{diag}\left(B_{1}, B_{2}\right)$, with respect to $H=N(A)^{\perp} \oplus N(A)$. Since $A B=\operatorname{diag}\left(A_{1} B_{1}, 0\right)$, we have that $A B$ is 
nilpotent if and only if $A_{1} B_{1}$ is nilpotent. Hence, without loss of generality, we may assume that $A$ is injective. Then $R(A)$ is dense, since $A$ is normal and injective. If there exists $n \in \mathbb{N}$ such that $(A B)^{n}=0$, then $(A B)^{n-1}=0$, since $R(A)$ and $R(B)$ are dense. By the same deduction, we can get $A B=0$. It is a contradiction.

Next, we turn to the study of properties of pairs of operators commuting up to a scalar factor with one of the operators having finite rank.

As in the proof of Theorem 2.5, if $A B=\lambda B A$ and $A$ is normal, then $N(A)$ and $\overline{R(A)}$ are reducing subspaces of $A$ and $B$. Thus $A$ and $B$ have forms $A=\operatorname{diag}\left(A_{1}, 0\right)$ and $B=\operatorname{diag}\left(B_{1}, B_{2}\right)$, with respect to $H=N(A)^{\perp} \oplus N(A)$. Suppose that $A$ has finite rank and $\left\{a_{1}, \cdots, a_{m}\right\}$ are the distinct nonzero eigenvalues of $A$. Then $A=\sum_{i=1}^{m} a_{i} P_{i}$, where $P_{i}$ is the orthogonal projection from $H$ onto $N\left(A-a_{i} I\right)$, for $1 \leq i \leq m$ and $P_{i} P_{j}=P_{j} P_{i}=0, i \neq j$. Denote $H_{i}=P_{i} H$. Then $A_{1}$ and $B_{1}$ have operator matrix forms $A_{1}=\operatorname{diag}\left(a_{1} I, \cdots, a_{m} I\right)$ and $B_{1}=\left(B_{i j}\right)_{m \times m}$, with respect to the space decomposition $N(A)^{\perp}=\bigoplus_{i=1}^{m} H_{i}$, respectively. With the symbols above, we have the following three results.

Proposition 2.6. Suppose that $A \in B(H)$ has finite rank and is normal. If $\sigma(A) \cap$ $\sigma(\lambda A)$ has no nonzero element, then there does not exist nonzero $B \in B(H)$ such that $A B=\lambda B A \neq 0$. If $\sigma(A) \cap \sigma(\lambda A)$ has nonzero elements, then there exists $B \in B(H)$ such that $A B=\lambda B A \neq 0$ if and only if either $m=1, \lambda=1$ or $m>1, \lambda \in \mathbb{C} \backslash\{0\}$.

Proof. If $A=0$, it is trivial. If $A \neq 0$, then we have $A B=\lambda B A \neq 0$ if and only if $A_{1} B_{1}=\lambda B_{1} A_{1} \neq 0$, since $A B=\operatorname{diag}\left(A_{1} B_{1}, 0\right)$. Hence, without loss of generality, we may assume that $A$ is injective. Also, $A B=\lambda B A$ if and only if $a_{i} B_{i j}=\lambda a_{j} B_{i j}$, for $1 \leq i, j \leq m$. If $\sigma(A) \cap \sigma(\lambda A)$ has no nonzero element, then $a_{i} \neq \lambda a_{j}$, for $i, j$. Hence there does not exist $B_{i j}$ such that $a_{i} B_{i j}=\lambda a_{j} B_{i j} \neq 0$, for $i, j$. Clearly, if $m=1$ and $\lambda=1$, then we have $A B=\lambda B A \neq 0$, for each $0 \neq B \in B(H)$. If $m>1$ and $\sigma(A) \cap \sigma(\lambda A)$ has nonzero elements, then there exists $1 \leq i, j \leq m$ such that $a_{i}=\lambda a_{j}$. Hence for each $B$ with $B_{i j} \neq 0$ and $B_{s t}=0, s \neq i, t \neq j$, we have $A B=\lambda B A \neq 0$. On the other hand, if $A B=\lambda B A \neq 0$ and $m=1$, then we have $\lambda=1$, since $\sigma(A) \cap \sigma(\lambda A)$ has nonzero element $a_{1}$.

If, with respect to a suitable decomposition of the space, $B$ has a form $B=$ $\bigoplus_{i=1}^{s} J_{k}^{(i)} \bigoplus_{i=s+1}^{s+t} M_{j_{i}}^{(i)}(\oplus 0) \oplus B_{2}$, then we call it a standard form, where the $k \times k$ block operator matrix $J_{k}^{(i)}$ and the $j_{i} \times j_{i}$ block operator matrix $M_{j_{i}}^{(i)}$ are defined by

$$
J_{k}^{(i)}=\left(\begin{array}{cccc}
0 & B_{12}^{(i)} & & 0 \\
& 0 & \ddots & \\
& & \ddots & B_{(k-1) k}^{(i)} \\
B_{k 1}^{(i)} & & & 0
\end{array}\right), \quad M_{j_{i}}^{(i)}=\left(\begin{array}{cccc}
0 & B_{12}^{(i)} & & 0 \\
& 0 & \ddots & \\
& & \ddots & B_{\left(j_{i}-1\right) j_{i}}^{(i)} \\
0 & & & 0
\end{array}\right) \text {, }
$$

$s, t \in \mathbb{N} \cup\{0\}, k, j_{i} \in \mathbb{N}$ and $B_{(. . .)}^{(.)}$are exactly the nonzero entries of $\left(B_{i j}\right)_{m \times m}$. In this case, if there exist $a^{(i)} \in \sigma(A) \backslash\{0\}, 1 \leq i \leq s+t$ with $a^{(p)} \neq a^{(l)}$ for $1 \leq p \neq l \leq s+t$, such that $A$ has a form $A=\bigoplus_{i=1}^{s} I_{k}^{(i)} \bigoplus_{i=s+1}^{s+t} N_{j_{i}}^{(i)} \oplus A^{\prime}, \lambda^{k}=1$ and $j_{i} \leq k$ for $s+1 \leq i \leq s+t$, where $A^{\prime}$ is a diagonal operator and the $k \times k$ block 
operator matrix $I_{k}^{(i)}$ and the $j_{i} \times j_{i}$ block operator matrix $N_{j_{i}}^{(i)}$ are defined by

$$
I_{k}^{(i)}=\left(\begin{array}{cccc}
a^{(i)} I & & & \\
& \lambda a^{(i)} I & & \\
& & \ddots & \\
& & & \lambda^{k-1} a^{(i)} I
\end{array}\right), \quad N_{j_{i}}^{(i)}=\left(\begin{array}{cccc}
a^{(i)} I & & & \\
& \lambda a^{(i)} I & & \\
& & \ddots & \\
& & & \lambda^{j_{i}-1} a^{(i)} I
\end{array}\right),
$$

respectively, then we say $A$ and $B$ are compatible.

Theorem 2.7. Suppose that $0 \neq A \in B(H)$ has finite rank and is normal. Then $A B=\lambda B A$ if and only if one of the following conditions holds:

(i) there exists a $B_{i i} \neq 0,1 \leq i \leq m, \lambda=1$ and $B=\operatorname{diag}\left(B_{11}, \cdots, B_{m m}, B_{2}\right)$;

(ii) $B_{i i}=0$, for $1 \leq i \leq m$, and $A$ and $B$ are compatible.

Proof. It is easy to show that (i) and (ii) imply $A B=\lambda B A$, respectively.

Conversely, from $A B=\lambda B A$, we have $a_{i} B_{i j}=\lambda a_{j} B_{i j}$. If there exists a $B_{i i} \neq 0$, for some $1 \leq i \leq m$, then $\lambda=1$ and $A B=B A$. Since $\left(A-a_{i} I\right) B=B\left(A-a_{i} I\right)$, by the Fuglede-Putnam Theorem, $H_{i}$ is a reducing subspace of $B$. Then $B_{i j}=$ $0, i \neq j$. Hence $B=\operatorname{diag}\left(B_{11}, \cdots, B_{m m}\right)$. If $B_{i i}=0$ for $1 \leq i \leq m$, then $A B=\lambda B A$ implies that $a_{i} B_{i j}=\lambda a_{j} B_{i j}$. Since $a_{i} \neq a_{j}, i \neq j$, we obtain that there is at most one nonzero entry in each row and column of $\left(B_{i j}\right)_{m \times m}$. It is easy to see that with respect to a suitable decomposition of the space, $B$ has a form $B=\bigoplus_{i=1}^{s} J_{k_{i}}^{(i)} \bigoplus_{i=s+1}^{s+t} M_{j_{i}}^{(i)} \oplus B_{2}$, where

$$
J_{k_{i}=}^{(i)}\left(\begin{array}{cccc}
0 & B_{12}^{(i)} & & 0 \\
& 0 & \ddots & \\
& & \ddots & B_{\left(k_{i}-1\right) k_{i}}^{(i)} \\
B_{k_{i} 1}^{(i)} & & & 0
\end{array}\right), \quad M_{j_{i}}^{(i)}=\left(\begin{array}{cccc}
0 & B_{12}^{(i)} & & 0 \\
& 0 & \ddots & \\
& & \ddots & B_{\left(j_{i}-1\right) j_{i}}^{(i)} \\
0 & & & 0
\end{array}\right) \text {, }
$$

$s, t \in \mathbb{N} \cup\{0\}, k_{i}, j_{i} \in \mathbb{N}$ and $B_{(., .)}^{(.)}$are exactly the nonzero entries of $\left(B_{i j}\right)_{m \times m}$. Also, simple computation, we can get that there exists $a^{(i)} \in \sigma(A) \backslash\{0\}, 1 \leq i \leq s+t$ with $a^{(p)} \neq a^{(l)}$ for $1 \leq p \neq l \leq s+t$ such that $A$ has a form $A=\bigoplus_{i=1}^{s} I_{k_{i}}^{(i)} \bigoplus_{i=s+1}^{s+t} N_{j_{i}}^{(i)} \oplus$ $A^{\prime}$, with respect to the same decomposition of the space, where $A^{\prime}$ is a diagonal operator and the $k_{i} \times k_{i}$ block operator matrix $I_{k_{i}}^{(i)}$ and the $j_{i} \times j_{i}$ block operator matrix $N_{j_{i}}^{(i)}$ are defined by

$$
I_{k_{i}}^{(i)}=\left(\begin{array}{cccc}
a^{(i)} I & & & \\
& \lambda a^{(i)} I & & \\
& & \ddots & \\
& & & \lambda^{k_{i}-1} a^{(i)} I
\end{array}\right), \quad N_{j_{i}}^{(i)}=\left(\begin{array}{cccc}
a^{(i)} I & & & \\
& \lambda a^{(i)} I & & \\
& & \ddots & \\
& & & \lambda^{j_{i}-1} a^{(i)} I
\end{array}\right) .
$$

We will show that if $s>1$, then $k_{1}=\cdots=k_{s}$. We assume that $k_{i}<k_{j}, 1 \leq i, j \leq s$; then $\lambda^{k_{i}}=1$ and $\lambda^{k_{j}}=1$. Hence $\lambda^{k_{j}-k_{i}}=1$. Therefore $a^{(i)}=\lambda^{k_{j}-k_{i}} a^{(i)}$. It is a contradiction with $a_{i} \neq a_{j}, i \neq j$. Hence $k_{1}=\cdots=k_{s}$. Let $k=k_{i}$; then, clearly we have $\lambda^{k}=1$ and the $j_{i} \leq k$, for $s+1 \leq i \leq s+t$. That is to say, $A$ and $B$ are compatible.

Remark. If $A$ is compact and normal, a result similar to Theorem 2.7 can be obtained by the same deduction. Its representation is more complex, and so it is omitted. 
For given $0 \neq A \in B(H)$, let $S_{A}^{\lambda}=\{B \in B(H): A B=\lambda B A\}$. Then $S_{A}^{\lambda}$ is a closed subspace of $B(H)$. If $\lambda^{n}=1$, for some $n \in \mathbb{N}$, then for each $B \in S_{A}^{\lambda}$, we have $B^{n+1} \in S_{A}^{\lambda}$. In fact, $A B^{n+1}=\lambda^{n+1} B^{n+1} A=\lambda B^{n+1} A$. If $H$ is finite dimensional, by Theorem 4.4.6 in [10, we have $S_{A}^{\lambda} \neq\{0\}$ if and only if $\sigma(A) \cap \sigma(\lambda A) \neq \phi$.

Proposition 2.8. Suppose that $H$ is finite dimensional and $0 \neq A \in B(H)$ is normal. Then

$$
\operatorname{dim} S_{A}^{\lambda}=(\operatorname{dim} N(A))^{2}+\sum_{1 \leq i, j \leq m} \operatorname{dim} H_{i} \operatorname{dim} H_{j} \psi(i, j),
$$

where $\psi(i, j)=\left\{\begin{array}{ll}1 & a_{i}=\lambda a_{j} \\ 0 & a_{i} \neq \lambda a_{j}\end{array}\right.$.

Proof. Suppose that $A$ is normal. Then $A B=\lambda B A$ if and only if $A_{1} B_{1}=\lambda B_{1} A_{1}$. Hence $\operatorname{dim} S_{A}^{\lambda}=\operatorname{dim} S_{A_{1}}^{\lambda}+(\operatorname{dim} N(A))^{2}$. Next, we assume that $A$ is injective. We will show that $\operatorname{dim} S_{A}^{\lambda}=\sum_{1 \leq i, j \leq m} \operatorname{dim} H_{i} \operatorname{dim} H_{j} \psi(i, j)$. In this case, $A B=\lambda B A$ if and only if $a_{i} B_{i j}=\lambda a_{j} B_{i j}$, for $1 \leq i, j \leq m$. If $a_{i} \neq \lambda a_{j}$, i.e. $\psi(i, j)=0$, for some $1 \leq i, j \leq m$, then $A B=\lambda B A$ implies $B_{i j}$ must be zero. If $a_{i}=\lambda a_{j}$, i.e. $\psi(i, j)=1$, for some $1 \leq i, j \leq m$, then for each $B_{i j} \in M_{\operatorname{dim} H_{i} \times \operatorname{dim} H_{j}}$, we have $a_{i} B_{i j}=\lambda a_{j} B_{i j}$. Thus $\operatorname{dim} S_{A}^{\lambda}=\sum_{1 \leq i, j \leq m} \operatorname{dim} H_{i} \operatorname{dim} H_{j} \psi(i, j)$.

\section{Sequential quantum measure}

We first prove a lemma.

Lemma 3.1. Suppose that $A$ is an injective positive operator and $B \in B(H)$ with dense range. If $A \circ B \in P(H)$, then $A B=B A=I$.

Proof. Since $R(A)$ is dense in $H$, we have that $R\left(A^{\frac{1}{2}}\right)$ is dense. That is, $\overline{R\left(A^{\frac{1}{2}}\right)}=$ $H$. Hence $\overline{R\left(B A^{\frac{1}{2}}\right)}=H$. This implies that $\overline{R\left(A^{\frac{1}{2}} B A^{\frac{1}{2}}\right)}=H$. By the assumption $A \circ B \in P(H)$, we have $A^{\frac{1}{2}} B A^{\frac{1}{2}}=I$. Therefore $R\left(A^{\frac{1}{2}}\right)=H$. This shows that $A$ is invertible. Hence $B=A^{-1}$, i.e., $A B=B A=I$.

If $A$ is positive and $B \in B(H)$, then $A$ and $B$ have operator matrices $A=$ $\operatorname{diag}\left(A_{1}, 0\right)$ and $B=\left(\begin{array}{ll}B_{11} & B_{12} \\ B_{21} & B_{22}\end{array}\right)$ with respect to the space decomposition $H=$ $\overline{R(A)} \oplus N(A)$, respectively. Since

$$
\left(\begin{array}{cc}
A_{1}^{\frac{1}{2}} & 0 \\
0 & 0
\end{array}\right)\left(\begin{array}{ll}
B_{11} & B_{12} \\
B_{21} & B_{22}
\end{array}\right)\left(\begin{array}{cc}
A_{1}^{\frac{1}{2}} & 0 \\
0 & 0
\end{array}\right)=\left(\begin{array}{cc}
A_{1}^{\frac{1}{2}} B_{11} A_{1}^{\frac{1}{2}} & 0 \\
0 & 0
\end{array}\right)
$$

$A \circ B \in P(H)$ is equivalent to $A_{1} \circ B_{11} \in P(\overline{R(A)})$. With the symbols above we have

Theorem 3.2. Suppose that $A \in B(H)$ is positive and that $B \in B(H)$ is selfadjoint. Then $N(A)$ is an invariant subspace of $B, N\left(\left.B\right|_{\overline{R(A)}}\right)$ is an invariant subspace of $A$, and $A \circ B \in P(H)$ if and only if $A B=B A$ and $\left.\left.A\right|_{\overline{R\left(\left.B\right|_{\overline{R(A)}}\right)}} \circ B\right|_{\overline{R\left(\left.B\right|_{\overline{R(A)}}\right)}} \in$ $P(\overline{R(B \mid \overline{R(A)})})$.

Proof. Suppose that $A \in B(H)$ is positive, $B \in B(H)$ is selfadjoint, $N(A)$ is an invariant subspace of $B, N\left(\left.B\right|_{\overline{R(A)}}\right)$ is an invariant subspace of $A$ and $A \circ$ $B \in P(H)$. Then we have $A=\operatorname{diag}\left(A_{1}, 0\right), B=\operatorname{diag}\left(B_{11}, B_{22}\right)$ and $A_{1} \circ B_{11} \in$ 
$P(\overline{R(A)})$, where $A_{1}=\left.A\right|_{\overline{R(A)}}, B_{11}=\left.B\right|_{\overline{R(A)}}$ and $B_{22}=\left.B\right|_{N(A)}$. Hence $B_{11} A_{1} B_{11}=$ $B_{11}$. If we consider the space decomposition $\overline{R(A)}=N\left(B_{11}\right) \oplus \overline{R\left(B_{11}\right)}$, we have $A_{1}=\operatorname{diag}\left(A_{11}, A_{22}\right)$ and $B_{11}=\operatorname{diag}\left(0, B_{11}^{\prime}\right)$. Hence $\left.B_{11} A_{1}\right|_{\overline{R\left(B_{11}\right)}}=I \overline{R\left(B_{11}\right)}$ and $B_{11}^{\prime} A_{22} B_{11}^{\prime}=B_{11}^{\prime}$. Then we have $A_{22} \circ B_{11}^{\prime} \in P\left(\overline{R\left(B_{11}\right)}\right)$. By Lemma 3.1, we have $A_{22} B_{11}^{\prime}=I_{\overline{R\left(B_{11}\right)}}$. Hence $A_{1} B_{11}=\left(\begin{array}{cc}0 & 0 \\ 0 & A_{22} B_{11}^{\prime}\end{array}\right)=\left(\begin{array}{cc}0 & 0 \\ 0 & I\end{array}\right)=B_{11} A_{1}$.

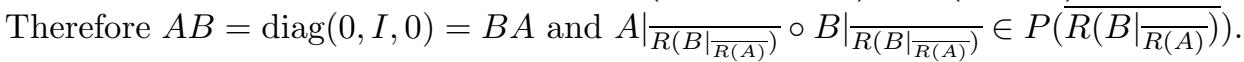

On the other hand, suppose that $A B=B A$ and $\left.\left.A\right|_{\overline{R\left(\left.B\right|_{\overline{R(A)}}\right.}} \circ B\right|_{\overline{R\left(\left.B\right|_{\overline{R(A)}}\right.}} \in$ $P\left(\overline{R\left(\left.B\right|_{\overline{R(A)}}\right)}\right)$. With respect to $H=\overline{R(A)} \oplus N(A), A$ and $B$ have operator matrices $A=\operatorname{diag}\left(A_{1}, 0\right)$ and $B=\left(\begin{array}{cc}B_{11} & B_{12} \\ B_{12}^{*} & B_{22}\end{array}\right)$, respectively. By the assumption, we have $A B=\left(\begin{array}{cc}A_{1} B_{11} & A_{1} B_{12} \\ 0 & 0\end{array}\right)=\left(\begin{array}{cc}B_{11} A_{1} & 0 \\ B_{12}^{*} A_{1} & 0\end{array}\right)=B A$. Hence $B_{12}=0$ and $A_{1} B_{11}=B_{11} A_{1}$. Therefore $N(A)$ is an invariant subspace of $B$. With respect to $\overline{R(A)}=N\left(B_{11}\right) \oplus \overline{R\left(B_{11}\right)}, A_{1}$ and $B_{11}$ have operator matrices $A_{1}=\left(\begin{array}{cc}A_{11} & A_{12} \\ A_{12}^{*} & A_{22}\end{array}\right)$ and $B_{11}=\operatorname{diag}\left(0, B_{11}^{\prime}\right)$, respectively. From $A_{1} B_{11}=B_{11} A_{1}$ we have $A_{12} B_{11}^{\prime}=$ 0 . Hence $A_{12}=0$ and $A \circ B=\operatorname{diag}\left(0, A_{22}^{\frac{1}{2}} B_{11}^{\prime} A_{22}^{\frac{1}{2}}, 0\right)$. Therefore $N\left(\left.B\right|_{\overline{R(A)}}\right)$ is an invariant subspace of $A$ and $A \circ B \in P(H)$, since $\left.\left.A\right|_{\overline{R\left(\left.B\right|_{\overline{R(A)}}\right)}} \circ B\right|_{\overline{R\left(\left.B\right|_{\overline{R(A)}}\right.}} \in$ $P\left(\overline{R\left(\left.B\right|_{\overline{R(A)}}\right)}\right)$.

If $A, B \in \varepsilon(H)$, then we can easily get that the part of the necessity condition of Theorem 3.2 is Theorem 1.3 in [9]. The virtue of our proof is that we can know the structure of $A, B$ and $A B$ clearly. One can use this method to prove Corollary 2.4, Theorem 2.5 and Theorem 2.6 in [9].

The following theorem is an answer to the question raised by S. Gudder and G. Nagy in 9], which is also a generalization of Theorem 2.6 (c) in [9]. This question was also independently answered by A. Gheondea and S. Gudder in [7. Though our proof is essentially the same as A. Gheondea and S. Gudder's, maybe our presentation of it is better and simpler. We still retain the proof here.

Theorem 3.3. If $A, B \in \varepsilon(H)$, then we have $A \circ B \geq B$ if and only if $A B=B A=$ $B$.

Proof. It is clear that $A B=B A=B$ implies $A \circ B \geq B$. Conversely, if

$$
A \circ B=\left(\begin{array}{cc}
A_{1}^{\frac{1}{2}} B_{11} A_{1}^{\frac{1}{2}} & 0 \\
0 & 0
\end{array}\right) \geq\left(\begin{array}{cc}
B_{11} & B_{12} \\
B_{12}^{*} & B_{22}
\end{array}\right)
$$

then $B_{12}=B_{22}=0$. Hence $B=\operatorname{diag}\left(B_{11}, 0\right)$ and $A_{1}^{\frac{1}{2}} B_{11} A_{1}^{\frac{1}{2}} \geq B_{11}$. This implies that $A_{1}^{\frac{n}{2}} B_{11} A_{1}^{\frac{n}{2}} \geq B_{11}$, for $n \in \mathbb{N}$. Suppose that $A_{1}=\int_{0}^{1} \lambda d E \lambda$ is the spectral calculus of $A_{1}$. Let $H_{1}=E(1)$ and $H_{2}=E([0,1))$. Then for each $x \in H_{2}$, we have $A_{1}^{\frac{n}{2}} x \rightarrow 0$ as $n \rightarrow \infty$. This implies $B_{11} x=0$, for $x \in H_{2}$. Hence $A=\operatorname{diag}\left(I, A_{1}^{\prime}, 0\right)$ and $B=\operatorname{diag}\left(B_{11}^{\prime}, 0,0\right)$, with respect to $H=H_{1} \oplus H_{2} \oplus N(A)$, where $A_{1}^{\prime}=\left.A\right|_{H_{2}}$ 
and $B_{11}^{\prime}=\left.B\right|_{H_{1}}$. Therefore,

$$
A B=\left(\begin{array}{ccc}
B_{11}^{\prime} & 0 & 0 \\
0 & 0 & 0 \\
0 & 0 & 0
\end{array}\right)=B A=B
$$

\section{ACKNOWLEDGEMENT}

We would like to thank Professor Huai-Xin Cao for his useful help. We also thank the referee for sending us the paper 7] of A. Gheondea and S. Gudder and giving many helpful suggestions which improved the presentation of the paper.

\section{REFERENCES}

[1] J. A. Brooke, P. Busch and B. Pearson, Commutativity up to a factor of bounded operators in complex Hilbert space, R. Soc. Lond. Proc. Ser. A Math. Phys. Eng. Sci., A458 (2002), no. 2017, 109-118. MR 2003b:47034

[2] P. Busch, Unsharp localization and causality in relativistic quantum theory, J. Phys., A32 (1999), no. 37, 6535-6546. MR 2001a:81093

[3] P. Busch, P. J. Lahti and P. Mittlestaedt, The quantum theory of measurement, SpringerVerlag, Berlin, 1991. MR 93m:81014

[4] P. Busch and J. Singh, Lüder theorem for unsharp quantum measurements, Phys. Lett., A249 (1998), 10-12.

[5] J. B. Conway, A course in functional analysis, Graduate Texts in Mathematics, No. 96, Springer-Verlag, New York, 1985. MR 86h:46001

[6] E. B. Davies, Quantum theory of open systems, Academic Press, London-New York, 1976. MR 58:8853

[7] A. Gheondea and S. Gudder, Sequential product of quantum effects, Proc. Amer. Math. Soc., to appear.

[8] S. Gudder and G. Nagy, Sequentially independent effects, Proc. Amer. Math. Soc., 130 (2002), no. 4, 1125-1130. MR 2002i:81014

[9] S. Gudder and G. Nagy, Sequential quantum measurements, J. Math. Phys., 42 (2001), 52125222. MR 2002h:81032

[10] R. A. Horn and C. R. Johnson, Matrix analysis, Cambridge Univ. Press, Cambridge, 1985. MR 87e:15001

[11] C. R. Putnam, Commutation properties of Hilbert space operators and related topics, Springer-Verlag, New York, 1967. MR 36:707

[12] J. von Neumann, Mathematical foundations of quantum mechanics, Princeton Univ. Press, Princeton, New Jersey, 1955. MR 16:654a

College of Mathematics and Information Science, Shaanxi Normal University, Xi'an 710062, P. R. CHINA

E-mail address: yangjia0426@sina.com

College of Mathematics and Information Science, Shaanxi Normal University, Xi'an 710062 , P. R. CHINA

E-mail address: hkdu@snnu.edu.cn 